\title{
On being yourself in different cultures: ideal and actual self-concept, autonomy support, and well-being in China, Russia, and the United States
}

\author{
Martin F. Lynch ${ }^{\mathrm{a} *}$, Jennifer G. La Guardia ${ }^{\mathrm{b}}$ and Richard M. Ryan ${ }^{\mathrm{c}}$ \\ ${ }^{a}$ Warner School of Education, University of Rochester, Rochester, NY, USA; ${ }^{b}$ University of Waterloo; ${ }^{c}$ University of \\ Rochester, Rochester, NY, USA
}

(Received 15 February 2008; final version received 20 December 2008)

\begin{abstract}
The present study used multilevel modeling and measures of the Big Five to test Rogers' prediction that discrepancies between ideal and actual self-concept would be negatively associated with well-being, and to test the prediction drawn from self-determination theory that partners' autonomy support would be associated with smaller discrepancies. Discrepancies and well-being were found to be negatively associated in samples from the USA, Russia, and China, but participants' actual self-concept was closer to their ideal when with autonomy supportive partners. Although there was some moderation by country membership, associations were in the same direction for all countries. Discussion focuses on the cultural and clinical implications.
\end{abstract}

Keywords: self-concept; autonomy support; culture; well-being; self-determination theory; Big Five

\section{Introduction}

The idea that people can have different views of themselves as they actually are and as they would ideally like to be, and that these self-concept discrepancies have implications for well-being, has a long tradition in humanistic (Rogers \& Dymond, 1954) and social-cognitive (Higgins, 1987) psychology, and indeed has been suggested by others as well (e.g., Lecky, 1945). However, to date little has been done to bring these assertions into line with contemporary thinking about personality and motivation, to test them using some of the newer statistical tools available to researchers, or to examine them cross-culturally. The present research used multilevel modeling to test whether ideal/actual discrepancies in self-concept, assessed using a measure of the Big Five, are associated with decrements to well-being in three countries that traditionally are thought to differ along the allocentricidiocentric dimension: China, Russia, and the United States. In addition, the study tested the proposition drawn from self-determination theory (SDT; Deci \& Ryan, 2000) that people should feel more free to pursue their personal ideal in relationships experienced as autonomy supportive. To set the framework for these predictions we briefly review two key perspectives on the association between ideal/actual self-concept discrepancies and well-being before presenting a selfdetermination theory account of the relation between the self and the social environment.

\section{Ideal and actual self-concept}

Rogers (1961) argued that self-concept plays an important role in the regulation of behavior by determining which aspects of experience are admissible to awareness and which aspects need to be 'repressed' so as to minimize conflict, whether with others or with oneself. Whether particular aspects of the self-concept are deemed acceptable or not is in part determined by the nature of one's interactions with others, including by the experience of being conditionally regarded by them. As important as one's current or actual selfconcept is, Rogers believed that people also have an ideal view of themselves, and that the gap between the current or actual view of self and the ideal view of self serves as an important gauge of self-esteem: the larger the gap, the lower one's self-esteem, while the closer people are to their ideal the better off they should be. He considered that awareness of a gap between one's current and ideal view of self often plays a major role in motivating people to seek counseling and psychotherapy. In a number of innovative studies, Rogers and his colleagues provided empirical support for a link between self-concept discrepancies and well-being (Rogers \& Dymond, 1954). Indeed, Rogers viewed a reduction in ideal/actual discrepancies to be an important therapeutic outcome, considering it to be an indication of positive personality change (Rogers, 1961). From a social-cognitive perspective, Higgins (1987, 1989) similarly argued and provided empirical

*Corresponding author. Email: mlynch@warner.rochester.edu 
evidence supporting the idea that when people experience a discrepancy between their actual self-concept and their ideal self-concept, they are likely to experience distress in the form of depressed affect. Accordingly, people are generally motivated to seek to reduce such ideal/actual self-concept discrepancies.

If it is the case that discrepancies in self-concept are associated with distress, as these and other researchers suggest, then it becomes important to identify factors in the interpersonal environment that may help to reduce such discrepancies. This possibility was not a central concern of Higgins' $(1987,1989)$ initial work. Rogers (1961) however argued that the therapeutic relationship, characterized by genuineness, empathy, and unconditional positive regard, could facilitate the reduction of ideal/actual discrepancies by creating an environment in which clients would feel safe to explore and integrate aspects of themselves that previously had lain outside their self-concept (or selfstructure), and therefore had been treated as off-limits or alien to the self. In this way, by exploring and integrating the various aspects of the self in the context of the therapeutic relationship, clients could feel more free to pursue their own ideal for the person they would like to be. Although his research focused on the therapeutic relationship, Rogers (1961) speculated that the same principles should apply to non-professional relationships as well. Building on these ideas, the present research employs the framework of self-determination theory (SDT) (Deci \& Ryan, 2000) to explore how an aspect of everyday relationships, namely autonomy support, may help to reduce ideal/actual discrepancies and improve well-being.

\section{The self in its interpersonal context: A self-determination theory view}

SDT shares common theoretical ground with interactionist (Magnusson \& Endler, 1977; Shoda, Mischel, \& Wright, 1994) and transactional (e.g., Srivastava, John, Gosling, \& Potter, 2003) theories in its suggestion that important processes in personality and development are influenced by both the individual and the social context. SDT further specifies, however, that in her interaction with the environment the person brings a set of innate, basic psychological needs for competence, relatedness, and autonomy which she seeks to fulfill, thereby activating organismic processes of growth and integration (Deci \& Ryan, 2000; Ryan \& Deci, 2000). The environment, in turn, can either provide or fail to provide opportunities to satisfy those needs. Satisfaction of the three needs in a particular context is associated with more internal motivation and greater well-being in that context. When, however, these needs are not satisfied people's motivation tends to become more external (oriented toward rewards and punishments) and their well-being declines (e.g., La Guardia, Ryan, Couchman, \& Deci, 2000; Lynch, Plant, \& Ryan, 2005).

SDT and Rogers' humanistic approach in fact share a common organismic metatheoretical underpinning (see Joseph \& Linley, 2004; Patterson \& Joseph, 2007). Accordingly, SDT's conceptualization of basic psychological needs offers a conceptually new yet theoretically consistent way to examine Rogers' suggestion that relationships can help or hinder people approaching their personal ideal. Of the three needs posited by SDT, autonomy may have the most relevance for the kind of personality processes that Rogers (1961) was pointing to (Markland, Ryan, Tobin, \& Rollnick, 2005). This is because autonomy, as conceptualized by SDT, concerns the need to feel oneself able to make personally meaningful choices, to take initiative, and to pursue personally held goals and ideals. Autonomy supportive partners provide opportunities for choice, initiative-taking, and personal goal-pursuit, avoid pressuring or controlling verbal or nonverbal behaviors, and generally engage in trying to understand the other person's internal frame of reference (Grolnick \& Ryan, 1989; Reeve, 2002; Reeve, Bolt, \& Cai, 1999; Ryan \& Lynch, 2003). A relationship that is experienced as supportive of the need for autonomy (in contrast to a relationship experienced as controlling or pressuring) should provide a likely context in which to feel free to pursue the self one would ideally like to be. Thus, it would be logical to expect that ideal/actual self-concept discrepancies should be smaller in autonomy supportive relationships. It should be noted that although historically research on the need for autonomy has focused on hierarchical relationships (e.g., between teacher and student, or doctor and patient), more recently attention has been given to the role of this need within close interpersonal relationships (e.g., Deci, La Guardia, Moller, Scheiner, \& Ryan, 2006; Patrick, Knee, Canevello, \& Lonsbary, 2007; Williams, Lynch, McGregor, Ryan, Sharp, \& Deci, 2006).

In this connection, we would argue that what has been called the current or actual self-concept may be more meaningfully explored within the context of one's current relationships. This is because we consider that actual self-concept properly speaking is typically instantiated (actualized) in some situational context, such as the context afforded by specific relationships. Without that situational anchor, we believe it would be more accurate to refer to 'global' or 'general' selfconcept. Hence, while previous researchers have investigated the importance of discrepancies between ideal and this general self-concept, we propose to explore discrepancies between the ideal and one's self-concept within a set of common, everyday relationships. We will refer to this latter as 'actual' 
self-concept, arguing that this formulation of the construct may have greater ecological validity than its more global counterpart and that it makes explicit the situational contexts that participants may implicitly call to mind when asked to think about how they view themselves 'in general.' An exciting aspect of this decision is that it moves us from more traditional between-persons analysis into the realm of withinperson processes. To accomplish this, we will use a data analytic technique known as multilevel modeling (MLM) (e.g., Fleeson, 2007).

\section{Big Five, self-concept, and culture}

The Big Five have emerged as perhaps the most reliable and universal way to measure individual differences in personality (John \& Srivastava, 1999; McCrae \& Costa, 2003), and considerable evidence has been amassed in support of the model's cross-cultural validity (e.g., McCrae \& Allik, 2002; McCrae et al., 2004; McCrae \& Terracciano, 2005; Schmitt, Allik, Mccrae, \& Benet-Martinez, 2007). Traits of course are typically thought of as being stable across situations and over time, but self-reported Big Five traits have been used as a way to measure self-concept and its variation (e.g., Roberts, Robins, Caspi, \& Trzesniewski, 2003; Sheldon, Ryan, Rawsthorne, \& Ilardi, 1997). The present study is the first to use the Big Five to test the prediction made by Rogers' (1961) and others (e.g., Higgins, 1987) concerning the relation of discrepancies in ideal and actual self-concept to well-being. As noted, no study to date has tested that prediction across cultures or by using a multilevel modeling approach to explore within-person associations. Further, while other studies have looked at mean levels of traits across countries (e.g., Schmitt et al., 2007), none to date has looked at trait ideals, that is, how the traits are valued in different cultures. Finally, the present research situates these issues within the motivational framework provided by SDT. The present research thus aims to provide a cross-cultural test of the relation of self-concept discrepancies to wellbeing, to bring earlier theories about self-concept discrepancies into line with contemporary thinking about personality and motivation, and to increase our understanding of how the traits are valued in different cultures.

A major hypothesis of the present research is that being with autonomy supportive partners should be associated with smaller ideal/actual discrepancies in self-concept. SDT argues that autonomy is a basic psychological need that is innate and therefore universal across cultures. This claim is controversial. An argument could be made that support for autonomy should only be important in cultures in which autonomy is explicitly valued (e.g., Markus \&
Kitayama, 1991), an argument which has been called the cultural 'match hypothesis.' Evidence for the role of autonomy across cultures has begun to emerge (Chirkov, Ryan, \& Willness, 2005; Jang, Reeve, Ryan, \& Kim, in press; Rudy, Sheldon, Awong, \& Tan, 2007; Vansteenkiste, Zhou, Lens, \& Soenens, 2005). The present research continues the empirical investigation of SDT's claim that autonomy is a basic psychological need, and that its support in interpersonal relationships should therefore be important across cultures. If the association between autonomy support and selfconcept discrepancies is moderated by country membership (such that autonomy support is related to selfconcept discrepancies in opposite directions depending on the country, or is related to outcomes in some countries but not in all), this would lend support to the cultural match hypothesis. If country membership does not alter the presence or direction of an association, this would lend support to the self-determination theory perspective.

\section{The present study}

The present study applied an SDT perspective to examine the relation between perceived autonomy support and self-concept. First, using a measure of the Big Five, the study revisited predictions made by Rogers (Rogers \& Dymond, 1954) and others (e.g., Higgins, 1987) regarding the association between ideal/actual self-concept discrepancies and well-being, as an important confirmation that the general hypothesis would hold when self-concept was measured with the Big Five, that it would hold across different cultural groups, and that it could be appropriately tested using this new multilevel modeling approach. Second, the study tested the prediction drawn from SDT that being with autonomy supportive partners should be associated with smaller ideal/actual discrepancies. Third, the generalizability of the SDT-based prediction on the role of autonomy support was tested among participants from three countries traditionally representing different locations along the cultural continuum of allocentrism-idiocentrism: China, Russia, and the United States. Country membership was used to test whether culture moderates the relation between self-concept discrepancies and wellbeing and between self-concept discrepancies and autonomy support. Based on SDT, our main prediction in this regard was that country membership would not moderate the relation between autonomy support and self-concept discrepancies, that is, that autonomy support would be negatively associated with ideal/ actual self-concept discrepancies, regardless of country membership. These associations were tested using multilevel modeling (MLM). 


\section{Method}

\section{Participants and procedures}

Participants were 642 college students drawn from three nations: 205 from a northeastern US university (65.4\% female; age $M=19.66, S D=1.99), 192$ from a university in European Russia (81.8\% female; age $M=18.99, S D=1.39$ ), and 245 from a university in the Shandong region in China $(68.6 \%$ female; age $M=20.71, S D=1.07)$. Participants volunteered for the study, receiving course credit or a small monetary compensation. Participants completed measures in small groups ( $<15$ students) over the course of two sessions, one week apart from each other.

\section{Measure translations}

To ensure fidelity of measures, translations and backtranslations were performed by persons highly fluent in either English and Russian or English and Chinese. Discrepancies were discussed and resolved by the translators and the first author. In addition, construct comparability across samples was statistically tested (see below).

\section{Measures: Session 1}

Ideal Big Five self-concept. To measure ideal Big Five self-concept, participants rated a set of 30 trait adjectives (Sheldon et al., 1997) on a scale of 1 (Not at all) to 7 (Very much). Participants first rated each adjective in terms of how they saw themselves 'in general,' and then contrasted that with how they ideally saw themselves. For the ideal adjective ratings, participants were given the instruction, 'Think of the attributes or characteristics you would ideally like to have - the type of person you wish, desire, or hope to be. Regardless of other people's opinions, these are the attributes that you feel are a reflection of how you would be ideally.' The phrase, 'regardless of other people's opinions,' was included in order to increase the likelihood that participants' ideal ratings would reflect personally held values rather than socially desirable trait expressions. Then they were provided the stem, 'Ideally, I would like to see myself as someone who is,' followed by each of the Big Five adjectives. Ideal self-concept scores were the average of the six items for each subscale (Extraversion, Neuroticism, Agreeableness, Openness to Experiences, and Conscientiousness). For extraversion, internal consistency alphas were $0.67,0.65$, and 0.68 for the US, Russian, and Chinese samples, respectively; $0.79,0.52$, and 0.67 for neuroticism; $0.72,0.74$, and 0.73 for agreeableness; $0.71,0.70$, and 0.70 for conscientiousness; and $0.65,0.64$, and 0.72 for openness to experience. General self-concept scores were not further used in the present study.

\section{Measures: Session 2}

Measures in Session 2 focused on within-person variations across six target relationships: Mother, Father, Best Friend, Romantic Partner, Roommate, and a self-selected Teacher ('please think about one of your current teachers, preferably the teacher with whom you currently have most contact'). The 'teacher' target was included so that a potentially hierarchicalsubordinate relationship would be assessed along with parental and peer relationships, and so that temporary as well as more lasting relationships would be included. Each relationship was presented in a separate section of the survey, and the order of presentation was counterbalanced across participants using a Latin square design.

\section{Perceived autonomy support}

Participants rated the perceived autonomy supportiveness of each partner using 7 items adapted from two existing scales: the Basic Need Satisfaction in Relationships Scale (BPNS-R) (La Guardia et al., 2000), and the Health Care Climate Questionnaire (HCCQ), originally validated by Williams and Deci (2001) to assess health care providers' autonomy support. The latter has been adapted for use in numerous settings, including the assessment of autonomy support from important others (e.g., Williams et al., 2006). Items adapted from the BPNS-R began with the stem, 'When I am with my [partner]' and included: 'I feel free to be who I am,' 'I have a say in what happens, and can voice my opinion,' and 'I feel controlled and pressured to be certain ways' (reversed). Items from the HCCQ included: 'I feel controlled by my [partner]' (reversed), 'I am not able to by myself with my [partner]' (reversed), 'My [partner] listens to my thoughts and ideas,' and 'My [partner] tries to understand how I see things.' Together these items tap various aspects of autonomy support as conceptualized within SDT, including the presence or absence of interpersonal pressure and control, and taking the other person's internal frame of reference. Items were rated on a 7-point scale from 1 (Strongly Disagree) to 7 (Strongly Agree). The mean of the seven items represents the perceived autonomy support score for that relationship. Alphas ranged from 0.67 to 0.71 , 0.59 to 0.77 , and 0.80 to 0.93 for Russian, Chinese, and US samples, respectively.

\section{Actual Big Five self-concept (within relationships)}

Participants rated themselves on each of the $30 \mathrm{Big}$ Five items as they experienced them within each target relationship. Again, we refer to this as actual selfconcept because we consider that actual self-concept properly speaking is typically instantiated (actualized) in some situational context, such as the context 
afforded by specific relationships. For the US sample, alphas ranged from 0.70 to 0.85 for extraversion across the six relationships, 0.74 to 0.85 for neuroticism, 0.77 to 0.85 for agreeableness, 0.75 to 0.83 for conscientiousness, and 0.70 to 0.80 for openness. For the Chinese sample, the ranges were 0.72 to 0.78 (extraversion), 0.54 to 0.75 (neuroticism), 0.70 to 0.78 (agreeableness), 0.63 to 0.77 (conscientiousness), and 0.43 to 0.77 (openness). For the Russian sample, these ranges were 0.54 to 0.68 (extraversion), 0.55 to 0.70 (neuroticism), 0.70 to 0.83 (agreeableness), 0.66 to 0.80 (conscientiousness), and 0.69 to 0.82 (openness).

\section{Relational well-being}

To test the prediction that ideal/actual discrepancies would be related to well-being within relationships, four indicators of well-being were assessed within each of the target relationships: satisfaction, vitality, and positive and negative affect. First, participants rated their satisfaction within each relationship, on a 7-point Likert-type scale $(1=$ not at all, $7=$ very much $)$, using the item 'How satisfied are you within this relationship?' Second, they were asked to rate their experience of vitality within each specific relationship using the 6-item version of the Subjective Vitality scale (Ryan \& Frederick, 1997) validated by Bostic, Rubio, and Hood (2000). Sample items include 'When I am with my mother, I feel alive and vital' and 'When I am with my mother, I have energy and spirit,' rated on a 7-point scale ranging from 'not at all' (1) to 'very much' (7). Finally, positive and negative affect, as experienced within each relationship, were assessed using the 20item PANAS, rated on a 7-point scale ranging from 'not at all' (1) to 'very much' (7) (Watson, Clark, \& Tellegen, 1988). Alphas for vitality within the six relationships ranged from 0.92 to 0.96 (USA), 0.93 to 0.96 (Russia), and 0.70 to 0.80 (China). Alphas for positive affect ranged from 0.88 to 0.95 (USA), 0.76 to 0.82 (Russia), and 0.74 to 0.84 (China); those for negative affect ranged from 0.88 to 0.94 (USA), 0.85 to 0.91 (Russia), and 0.84 to 0.91 (China). Across participants, absolute values of correlations among well-being indicators ranged from 0.43 to 0.82 for the US sample; 0.28 to 0.65 for the Russian sample; and 0.09 to 0.67 for the Chinese sample, all $p \mathrm{~s}<0.01$. Accordingly, a composite well-being index was created by summing the standardized scores of the well-being scales, with negative affect reversed.

\section{General analytic procedures}

\section{Construct comparability}

Establishing the comparability of constructs when examining between-groups differences is a critical issue in cross-cultural research (Cheung \& Rensvold, 2000; Little, Lindenberger, \& Nesselroade, 1999).
Following recommendations by Byrne (2001, 2002) and Little (1997, 2000), structural equation modeling was used to test for factorial invariance and measurement comparability across groups. Data for all scales demonstrated adequate fit, allowing for hypothesis testing using MLM.

\section{Multilevel modeling}

MLM (Fleeson, 2007) was used in order to assess the within-person process governing the relation between explanatory and outcome variables across six different relationship targets. Note that in this approach, 'within-person' means that both the explanatory variables (e.g., perceived autonomy support) and the outcome variable (e.g., well-being) are allowed to vary within each participant across their particular relationship targets. In addition, MLM permits a test of the possibility that between-person differences in the relationship between variables are not due to chance, denoted by the standard deviation on the main effect: in other words, the association between explanatory and outcome variables may be stronger or weaker in some participants compared to others. MLM requires that data be restructured so that cases are represented by multiple rows; in the present research, rows represented relationships with the six partner targets. MLM was conducted by means of the mixed models linear program in SPSS 15 (Fleeson, 2007). All continuous, within-person, situational or 'level 1' explanatory variables (e.g., autonomy support) were mean-centered within each person in order to account for between-person variance and ensure that results reflected the postulated within-person process. All participants had between three and six partners for whom they provided data. Cases for which there were fewer than three relationship targets were not included in these analyses. This resulted in excluding two cases from the US data, and five cases from the Chinese data. Initial analysis showed that key associations were not moderated by sex; accordingly, reported results collapse across sex.

Additional considerations. For the primary analyses the present research adopted the convention of comparing ideal and actual self-concept scores by computing the absolute difference between them. This allowed a more rigorous test of Rogers' (Rogers \& Dymond, 1954) hypothesis by including the possibility that overshooting one's ideal would be potentially as detrimental to well-being as falling short of it.

\section{Results}

\section{Preliminary analyses}

Comparing ideal and actual Big Five self-concept

As an initial test of whether ideal and actual selfconcept actually differed, paired sample $t$-tests were 
Table 1. Means for traits in the ideal and actual (relationship-specific) self-concept in China, Russia, and the United States.

\begin{tabular}{|c|c|c|c|c|c|c|c|c|c|c|c|c|c|c|}
\hline \multirow[b]{2}{*}{ Country } & \multicolumn{2}{|c|}{ Ideal } & \multicolumn{2}{|c|}{ Mother } & \multicolumn{2}{|c|}{ Father } & \multicolumn{2}{|c|}{ Best Friend } & \multicolumn{2}{|c|}{$\begin{array}{c}\text { Romantic } \\
\text { Partner }\end{array}$} & \multicolumn{2}{|c|}{ Roommate } & \multicolumn{2}{|c|}{ Teacher } \\
\hline & Mean & $S D$ & Mean & $S D$ & Mean & $S D$ & Mean & $S D$ & Mean & $S D$ & Mean & $S D$ & Mean & $S D$ \\
\hline \multicolumn{15}{|l|}{ China } \\
\hline Extraversion & $5.34_{\mathrm{ab}}$ & 0.97 & $5.19_{\mathrm{ad}}$ & 1.18 & $4.79 \mathrm{e}$ & 1.23 & $5.44_{\mathrm{b}}$ & 1.07 & $4.80_{\mathrm{ef}}$ & 1.24 & $5.07 \mathrm{df}$ & 1.08 & $4.34_{c}$ & 1.12 \\
\hline Neuroticism & $2.10_{\mathrm{a}}$ & 0.95 & $2.62_{\mathrm{e}}$ & 1.10 & $2.72_{b}$ & 1.17 & $2.46_{\mathrm{f}}$ & 0.94 & $3.16_{\mathrm{bgh}}$ & 1.13 & $2.95_{\mathrm{cg}}$ & 1.11 & $3.11_{\mathrm{dh}}$ & 1.24 \\
\hline Agreeableness & $5.73_{\mathrm{a}}$ & 0.87 & $4.94_{b}$ & 1.08 & $4.94_{\mathrm{b}}$ & 1.13 & $5.48_{\mathrm{c}}$ & 0.92 & $4.81_{\mathrm{bd}}$ & 1.16 & $5.12_{\mathrm{d}}$ & 1.02 & $4.98_{\mathrm{b}}$ & 1.06 \\
\hline Conscientiousness & $5.85_{\mathrm{a}}$ & 0.89 & $4.74_{\mathrm{d}}$ & 1.09 & $4.84_{\mathrm{bd}}$ & 1.02 & $5.30_{\mathrm{e}}$ & 0.94 & $4.65_{\mathrm{bdf}}$ & 1.17 & $5.07_{\mathrm{cf}}$ & 1.03 & $5.00_{\mathrm{cf}}$ & 1.00 \\
\hline Openness & $5.54_{\mathrm{a}}$ & 1.00 & $4.57_{\mathrm{cd}}$ & 1.21 & $4.59 \mathrm{~cd}$ & 1.13 & $4.88_{\mathrm{e}}$ & 1.03 & $4.47_{\mathrm{bcd}}$ & 1.20 & $4.61_{\mathrm{d}}$ & 1.14 & $4.37_{\mathrm{bc}}$ & 1.19 \\
\hline \multicolumn{15}{|l|}{ Russia } \\
\hline Extraversion & $4.96_{\mathrm{a}}$ & 0.63 & $5.14_{c}$ & 0.86 & $4.96_{\mathrm{a}}$ & 0.98 & $5.54_{d}$ & 0.87 & $5.13_{\mathrm{cd}}$ & 0.81 & $4.97 \mathrm{ad}$ & 0.92 & $4.34_{b}$ & 0.79 \\
\hline Neuroticism & $1.85_{\mathrm{a}}^{\mathrm{a}}$ & 0.59 & $2.46_{\mathrm{c}}$ & 0.74 & $2.61_{\mathrm{d}}$ & 0.91 & $2.27 \mathrm{e}$ & 0.74 & $2.34_{\mathrm{ce}}$ & 0.73 & $2.68_{\mathrm{d}}$ & 0.86 & $3.01_{b}$ & 0.82 \\
\hline Agreeableness & $5.63_{\mathrm{a}}$ & 0.80 & $5.30_{\mathrm{c}}$ & 0.92 & $5.03_{\mathrm{d}}$ & 1.08 & $5.44_{\mathrm{c}}$ & 0.77 & $5.43_{\mathrm{c}}$ & 0.83 & $4.70_{\mathrm{b}}$ & 1.05 & $4.70_{\mathrm{b}}$ & 0.83 \\
\hline Conscientiousness & $6.17_{\mathrm{a}}$ & 0.67 & $5.13_{\mathrm{c}}$ & 0.89 & $5.12_{\mathrm{bc}}$ & 0.82 & $5.07_{\mathrm{bc}}$ & 0.93 & $5.36_{\mathrm{d}}$ & 0.88 & $4.94_{b}$ & 0.92 & $5.32_{\mathrm{d}}$ & 0.88 \\
\hline Openness & $4.77 \mathrm{a}$ & 0.87 & $4.59_{\mathrm{d}}$ & 1.03 & $4.42_{\mathrm{e}}$ & 1.11 & $5.24_{\mathrm{f}}$ & 1.05 & $5.15_{\mathrm{f}}$ & 0.91 & $4.47_{\mathrm{bde}}$ & 1.30 & $4.16_{\mathrm{c}}$ & 1.06 \\
\hline \multicolumn{15}{|l|}{ United States } \\
\hline Extraversion & $5.65_{\mathrm{af}}$ & 0.71 & $5.42_{\mathrm{ac}}$ & 1.17 & $5.12_{\mathrm{d}}$ & 1.17 & $6.05_{\mathrm{e}}$ & 0.85 & $5.75_{\mathrm{f}}$ & 0.82 & $5.28_{\mathrm{cd}}$ & 1.22 & $4.35_{\mathrm{g}}$ & 1.10 \\
\hline Neuroticism & $1.54_{\mathrm{a}}$ & 0.64 & $2.63_{\mathrm{c}}$ & 1.09 & $2.65_{\mathrm{c}}$ & 1.10 & $2.00_{\mathrm{d}}$ & 0.81 & $2.28_{\mathrm{e}}$ & 0.88 & $2.65_{\mathrm{c}}$ & 1.12 & $2.60_{\mathrm{c}}^{\circ}$ & 0.88 \\
\hline Agreeableness & $6.29 \mathrm{a}$ & 0.62 & $5.20_{\mathrm{b}}$ & 0.98 & $5.13_{\mathrm{b}}$ & 1.00 & $5.81_{\mathrm{c}}$ & 0.74 & $5.77_{\mathrm{c}}$ & 0.79 & $5.41_{\mathrm{d}}$ & 0.93 & $5.52_{\mathrm{d}}$ & 0.83 \\
\hline Conscientiousness & $6.20 \mathrm{a}$ & 0.67 & $5.07_{\mathrm{b}}$ & 1.04 & $5.12_{\mathrm{b}}$ & 0.96 & $4.89_{\mathrm{c}}$ & 1.08 & $5.11_{\mathrm{b}}$ & 0.94 & $5.09 \mathrm{~b}$ & 1.01 & $5.63_{\mathrm{d}}$ & 0.94 \\
\hline Openness & $5.97_{\mathrm{a}}$ & 0.67 & $4.65_{\mathrm{b}}$ & 1.12 & $4.51_{\mathrm{b}}$ & 1.06 & $5.29_{\mathrm{c}}$ & 0.94 & $5.28_{\mathrm{c}}$ & 0.88 & $4.65_{\mathrm{b}}$ & 1.14 & $4.60_{\mathrm{b}}$ & 1.05 \\
\hline
\end{tabular}

Note: Subscripts denote comparisons within a row. Means sharing a common subscript do not differ $(p>0.05)$.

performed within each country (Table 1). There were only five cases in which the ideal did not significantly differ from the actual (relationship-specific) selfconcept score, each involving extraversion. In China, this lack of discrepancy was noted for the relationship with Best Friend; in Russia, for Father and Roommate; and in the United States, for Mother and Romantic Partner. In each of these relationships, participants reported experiencing their self-endorsed ideal level in their actual self-concept for extraversion. There were five cases in which participants' actual selfconcept scores significantly surpassed their ideal: in Russia, this occurred for extraversion (with Mother, Best Friend, and Romantic Partner) and openness (with Best Friend); in the United States, this occurred for extraversion (with Best Friend). In all other cases, actual self-concept levels were lower than the respective ideal levels, with the exception of neuroticism (for which the ideal levels were lower than actual levels). This finding is noteworthy in that it demonstrates that the Big Five are similarly valued by participants in three different countries: people wished to see themselves for example as more agreeable and less neurotic than they actually did see themselves.

\section{Comparing autonomy support across relationships}

Paired sample $t$-tests were used to make comparisons among mean levels of autonomy support within relationships (Table 2). In each country, participants identified their best friend as their most autonomy supportive partner (in China this designation was shared with mother). In all three countries, teacher was rated as the least autonomy supportive partner.

\section{Primary analyses \\ Well-being and ideal/actual self-concept discrepancies: moderation by country}

To test the hypothesis that greater absolute discrepancies between ideal and actual self-concept would be associated with decrements to well-being, data for the three countries were combined and an MLM analysis was conducted using the mixed models linear program in SPSS 15, with ideal/actual discrepancies (centered within each participant) for one of the five traits entered as the level 1 explanatory variable and the combined relational well-being scores as the outcome. In addition, country membership (entered as a factor, with $1=$ United States, $2=$ Russia, $3=$ China) was included as a level 2 explanatory variable. ${ }^{1}$ Thus, the analysis was run 5 times ( 5 ideal/actual self-concept discrepancies, one possible moderator).

As shown in Table 3, unstandardized betas indicate that absolute discrepancies between ideal and actual self-concept were significantly and negatively associated with relational well-being across samples, providing support for predictions made by Rogers (Rogers \& Dymond, 1954) and Higgins (1987). There were no main effects by country. There were, however, several significant interactions. The interactions indicated 
Table 2. Perceived autonomy support within relationships in China, Russia, and the United States.

\begin{tabular}{|c|c|c|c|c|c|c|c|c|c|c|c|c|}
\hline \multirow[b]{2}{*}{ Country } & \multicolumn{2}{|c|}{ Mother } & \multicolumn{2}{|c|}{ Father } & \multicolumn{2}{|c|}{ Best Friend } & \multicolumn{2}{|c|}{ Romantic Partner } & \multicolumn{2}{|c|}{ Roommate } & \multicolumn{2}{|c|}{ Teacher } \\
\hline & Mean & $S D$ & Mean & $S D$ & Mean & $S D$ & Mean & $S D$ & Mean & $S D$ & Mean & $S D$ \\
\hline China & $5.22_{\mathrm{a}}$ & 1.12 & $4.95_{b}$ & 1.09 & $5.36_{\mathrm{a}}$ & 1.01 & $4.78_{\mathrm{bd}}$ & 1.12 & $4.82_{\mathrm{bd}}$ & 1.02 & 4.34 & 0.94 \\
\hline Russia & $5.39 \mathrm{a}$ & 0.95 & $5.08_{\mathrm{b}}$ & 1.00 & 5.90 & 0.81 & $5.47 \mathrm{a}$ & 0.88 & $5.11_{\mathrm{b}}$ & 1.04 & 4.33 & 1.00 \\
\hline US & $5.49_{\mathrm{a}}^{\mathrm{a}}$ & 1.31 & $5.23_{\mathrm{b}}$ & 1.43 & 6.23 & 0.89 & $5.97^{\circ}$ & 0.98 & $5.35_{\mathrm{ab}}$ & 1.31 & 4.94 & 1.03 \\
\hline
\end{tabular}

Note: Subscripts denote comparisons within a row. Means sharing a common subscript do not differ $(p>0.05)$.

Table 3. The within-person process relating ideal/actual self-concept discrepancies, country, and their interaction to relational well-being in United States, Russian, and Chinese samples combined.

\begin{tabular}{|c|c|c|}
\hline Explanatory variables & Typical individual & $S D$ across individuals \\
\hline Extraversion Discrepancy & $-0.23^{*}$ & $1.18 * *$ \\
\hline Country 1 & -0.10 & - \\
\hline Country 2 & -0.18 & - \\
\hline Interaction (Country $1 \mathrm{X}$ Extraversion Discrepancy) & $-0.56^{* *}$ & - \\
\hline Interaction (Country $2 \mathrm{X}$ Extraversion Discrepancy) & -0.19 & - \\
\hline Neuroticism Discrepancy & $-0.51 * *$ & $1.10 * *$ \\
\hline Country 1 & -0.11 & - \\
\hline Country 2 & -0.20 & - \\
\hline Interaction (Country $1 \mathrm{X}$ Neuroticism Discrepancy) & $-1.50 * *$ & - \\
\hline Interaction (Country $2 \mathrm{X}$ Neuroticism Discrepancy) & $-1.24 * *$ & - \\
\hline Agreeableness Discrepancy & $-0.73 * *$ & $1.01 * *$ \\
\hline Country 1 & -0.10 & - \\
\hline Country 2 & -0.18 & - \\
\hline Interaction (Country 1 X Agreeableness Discrepancy) & $-0.92 * *$ & - \\
\hline Interaction (Country 2 X Agreeableness Discrepancy) & $-0.50 * *$ & - \\
\hline Openness Discrepancy & $-0.62 * *$ & $1.13^{* *}$ \\
\hline Country 1 & -0.10 & - \\
\hline Country 2 & -0.18 & - \\
\hline Interaction (Country 1 X Openness Discrepancy) & $-0.99 * *$ & - \\
\hline Interaction (Country 2 X Openness Discrepancy) & -0.09 & - \\
\hline Conscientiousness Discrepancy & $-0.51 * *$ & $1.05^{* *}$ \\
\hline Country 1 & -0.09 & - \\
\hline Country 2 & -0.16 & - \\
\hline Interaction (Country $1 \mathrm{X}$ Conscientiousness Discrepancy) & 0.08 & - \\
\hline Interaction (Country $2 \mathrm{X}$ Conscientiousness Discrepancy) & -0.38 & - \\
\hline
\end{tabular}

Note: Coefficients for 'Typical individual' are unstandardized. Country $1=$ United States; Country $2=$ Russia; Country 3 (China) is treated as redundant in the analysis, meaning that results for Countries 1 and 2 are with reference to Country 3.

$* p<0.05 ; * * p<0.01$.

that, although larger discrepancies were associated with poorer well-being outcomes for the typical individual in all three countries, for extraversion, neuroticism, agreeableness, and openness these associations were stronger (more negative) for participants from the United States compared to those from China. The associations were stronger among Russian compared to Chinese participants for neuroticism and agreeableness. Table 4, which presents the results of the same MLM analyses conducted within samples, clarifies these findings, highlighting that coefficients were larger for particular country groups, as noted.

As previously indicated, MLM provides a test of the possibility that the within-person process differed significantly across individuals. The results of this test, denoted by the standard deviations in Tables 3 and 4, indicate that the process did differ across individuals, such that although larger discrepancies were associated with poorer well-being for the typical individual in all three countries, for some individuals the association was stronger or weaker, or may even have been reversed. The one exception was conscientiousness among US participants, for whom the process did not differ significantly across individuals.

Taken together, results supported the hypothesis, based on previous work (Higgins, 1987; Rogers \& Dymond, 1954), that greater ideal/actual self-concept discrepancies would be associated with 
Table 4. The within-person process relating ideal/actual self-concept discrepancies to relational well-being in United States, Russian, and Chinese Samples.

\begin{tabular}{lll}
\hline Explanatory variables & $\begin{array}{c}\text { Typical } \\
\text { individual }\end{array}$ & $\begin{array}{c}S D \text { across } \\
\text { individuals }\end{array}$ \\
\hline United States & & \\
Extraversion Discrepancy & $-0.76^{* *}$ & $1.58^{* *}$ \\
Neuroticism Discrepancy & $-1.99^{* *}$ & $1.28^{* *}$ \\
Agreeableness Discrepancy & $-1.62^{* *}$ & $0.85^{* *}$ \\
Openness Discrepancy & $-1.58^{* *}$ & $1.42^{* *}$ \\
Conscientiousness Discrepancy & $-0.42^{*}$ & 0.84 \\
Russia & & \\
Extraversion Discrepancy & $-0.42^{*}$ & $0.97^{*}$ \\
Neuroticism Discrepancy & $-1.75^{* *}$ & $1.12^{* *}$ \\
Agreeableness Discrepancy & $-1.21^{* *}$ & $1.30^{* *}$ \\
Openness Discrepancy & $-0.75^{* *}$ & $1.03^{* *}$ \\
Conscientiousness Discrepancy & $-0.93^{* *}$ & $1.43^{* *}$ \\
China & & \\
Extraversion Discrepancy & $-0.27^{* *}$ & $0.89^{* *}$ \\
Neuroticism Discrepancy & $-0.50^{* *}$ & $0.81^{* *}$ \\
Agreeableness Discrepancy & $-0.72^{* *}$ & $0.85^{* *}$ \\
Openness Discrepancy & $-0.66^{* *}$ & $0.97^{* *}$ \\
Conscientiousness Discrepancy & $-0.52^{* *}$ & $0.84^{* *}$ \\
\hline
\end{tabular}

Note: Coefficients for 'Typical individual' are unstandardized.

$* p<0.05 ; * p<0.01$.

poorer well-being. This finding held in all three samples. In addition to providing a first test of the self-concept hypothesis using multilevel modeling, the present findings thus provide the first cross-cultural support for the predictions of these earlier researchers.

\section{Perceived autonomy support and ideal/actual self- concept discrepancies: Moderation by country}

The central prediction of the present research, based on SDT, was that greater autonomy support within one's relationships would be associated with smaller discrepancies between ideal and actual self-concept. To test this, data for the three countries were combined and an MLM analysis was conducted using the mixed models linear program in SPSS 15, with perceived autonomy support (centered within each participant) entered as the level 1 explanatory variable and ideal/ actual discrepancies for one of the Big Five as the outcome. To test for moderation, country membership (entered as a factor, with $1=$ United States, $2=$ Russia, $3=$ China) was included in the analysis. Thus, the analysis was run 5 times (5 ideal/actual discrepancies, one possible moderator).

The results are shown in Table 5. Unstandardized betas indicate that the experience of perceived autonomy support within relationships was associated with smaller discrepancies between one's ideal and actual self-concept for all of the Big Five (the association was marginally significant for conscientiousness). There were some main effects of country membership, such that the effect of being Russian on ideal/actual discrepancies was negative for all of the Big Five except for conscientiousness (Russians tended to have smaller discrepancies, compared to participants from China). Significant interactions indicate that the association between autonomy support and decreased discrepancies was greater for US than for Chinese participants for extraversion, neuroticism, agreeableness, and openness, but that it was marginally greater for Chinese than US participants for conscientiousness. Similarly, interactions indicate that the association between autonomy support and decreased discrepancies was greater for Russian than for Chinese participants for neuroticism and agreeableness, but was marginally stronger for Chinese than for Russian participants for extraversion. Table 6, presenting the within-country associations between autonomy support and ideal/actual discrepancies, clarifies the direction of these interactions as noted above. (Autonomy support was not associated with extraversion discrepancies among Russians, but it should be recalled that Russian participants attained their ideal on this trait within several relationships.)

The within-person process differed significantly across individuals in a number of cases, as indicated by the standard deviations in Tables 5 and 6 . Although experiencing more autonomy support was associated with smaller ideal/actual discrepancies for the typical individual in all three countries, for some individuals the association was weaker and for others it was stronger. The one exception was conscientiousness among Russian participants, for whom the process did not differ significantly across individuals.

Taken together, these results indicate that, for the typical individual, being with autonomy supportive partners was associated with thinking of oneself as being closer to one's ideal on each of the Big Five except conscientiousness in the US sample, on all but conscientiousness and extraversion in the Russian sample, and on all of the Big Five in the Chinese sample. Although there was some moderation of the strength of the association by country membership, the associations were in the same direction in each country, providing support for the SDT prediction that the experience of autonomy support remains important across countries.

\section{Autonomy support and relational well-being}

SDT predicts that if autonomy is a basic psychological need, then experiencing support for autonomy should be beneficial for well-being across cultures (Ryan \& Deci, 2000). Accordingly, an MLM analysis was conducted on the merged data set with autonomy support (centered within each individual) as the level 1 explanatory variable, country as the level 2 variable (factor), and relational well-being as the outcome. 
Table 5. The within-person process relating perceived autonomy support, country, and their interaction to ideal/actual self-concept discrepancies in United States, Russian, and Chinese samples combined.

\begin{tabular}{|c|c|c|}
\hline Explanatory variables (sorted by outcome) & Typical individual & $S D$ across individuals \\
\hline \multicolumn{3}{|l|}{ Extraversion Discrepancy } \\
\hline Autonomy Support & $-0.13 * *$ & $0.30 * *$ \\
\hline Country 1 & -0.06 & - \\
\hline Country 2 & $-0.29 * *$ & - \\
\hline Interaction (Country 1 X Autonomy Support) & $-0.18 * *$ & - \\
\hline Interaction (Country $2 \mathrm{X}$ Autonomy Support) & $0.10 *$ & - \\
\hline \multicolumn{3}{|l|}{ Neuroticism Discrepancy } \\
\hline Autonomy Support & $-0.14 * *$ & $0.22 * *$ \\
\hline Country 1 & 0.06 & - \\
\hline Country 2 & $-0.16 * *$ & - \\
\hline Interaction (Country 1 X Autonomy Support) & $-0.28 * *$ & - \\
\hline Interaction (Country $2 \mathrm{X}$ Autonomy Support) & $-0.17 * *$ & - \\
\hline \multicolumn{3}{|l|}{ Agreeableness Discrepancy } \\
\hline Autonomy Support & $-0.10 * *$ & $0.22 * *$ \\
\hline Country 1 & -0.03 & - \\
\hline Country 2 & $-0.13 * *$ & - \\
\hline Interaction (Country 1 X Autonomy Support) & $-0.13 * *$ & - \\
\hline Interaction (Country $2 \mathrm{X}$ Autonomy Support) & $-0.13^{* *}$ & - \\
\hline \multicolumn{3}{|l|}{ Openness Discrepancy } \\
\hline Autonomy Support & $-0.15 * *$ & $0.22 * *$ \\
\hline Country 1 & 0.02 & - \\
\hline Country 2 & $-0.36^{* *}$ & - \\
\hline Interaction (Country $1 \mathrm{X}$ Autonomy Support) & $-0.21 * *$ & - \\
\hline Interaction (Country $2 \mathrm{X}$ Autonomy Support) & 0.06 & - \\
\hline \multicolumn{3}{|l|}{ Conscientiousness Discrepancy } \\
\hline Autonomy Support & $-0.05+$ & $0.15^{* *}$ \\
\hline Country 1 & 0.06 & - \\
\hline Country 2 & -0.07 & - \\
\hline Interaction (Country 1 X Autonomy Support) & $0.09 *$ & - \\
\hline Interaction (Country $2 \mathrm{X}$ Autonomy Support) & 0.02 & - \\
\hline
\end{tabular}

Note: Coefficients for 'Typical individual' are unstandardized. Country $1=$ United States; Country $2=$ Russia; Country 3 (China) is treated as redundant in the analysis, meaning that results for Countries 1 and 2 are with reference to Country 3.

$* p<0.05 ; * *<0.01 ;+p<0.09$.

Table 6. The within-person process relating autonomy support to ideal/actual self-concept discrepancies in United States, Russian, and Chinese Samples.

\begin{tabular}{lcc}
\hline Outcome variables (sorted by country) & Typical individual & $S D$ across individuals \\
\hline United States & & \\
Extraversion Discrepancy & $-0.31^{* *}$ & $0.38^{* *}$ \\
Neuroticism Discrepancy & $-0.42^{* *}$ & $0.28^{* *}$ \\
Agreeableness Discrepancy & $-0.23^{* *}$ & $0.20^{* *}$ \\
Openness Discrepancy & $-0.35^{* *}$ & $0.18^{* *}$ \\
Conscientiousness Discrepancy & 0.04 & $0.16^{*}$ \\
Russia & & $0.22^{* *}$ \\
Extraversion Discrepancy & -0.03 & $0.20^{* *}$ \\
Neuroticism Discrepancy & $-0.31^{* *}$ & $0.27^{* *}$ \\
Agreeableness Discrepancy & $-0.23^{* *}$ & $0.26^{* *}$ \\
Openness Discrepancy & $-0.09^{* *}$ & 0.11 \\
Conscientiousness Discrepancy & -0.02 & $0.30^{* *}$ \\
China & & $0.18^{* *}$ \\
Extraversion Discrepancy & $-0.13^{* *}$ & $0.18^{* *}$ \\
Neuroticism Discrepancy & $-0.15^{* *}$ & $0.21^{* *}$ \\
Agreeableness Discrepancy & $-0.10^{* *}$ & $0.19^{* *}$ \\
Openness Discrepancy & $-0.15^{* *}$ & \\
Conscientiousness Discrepancy & $-0.05+$ & \\
\hline
\end{tabular}

Note: Coefficients for 'Typical individual' are unstandardized.

$* p<0.05 ; * *<0.01 ;+p<0.09$. 
The unstandardized beta for the process relating autonomy support to relational well-being was $\mathrm{b}=0.85, p<0.01$. For the typical individual, being with autonomy supportive partners was associated with greater well-being within relationships. The process differed significantly across individuals $(S D=$ $0.61, p<0.01$ ), indicating the relation was weaker or stronger for some. Although there were no significant main effects for country $(p s>0.2)$, there were two significant interactions such that the associations were stronger for US $(b=0.64, p<0.01)$ and for Russian $(b=0.22, p=0.05)$ than for Chinese participants.

Previous analyses demonstrated a link in the present samples between ideal/actual discrepancies and well-being, and between autonomy support and ideal/actual discrepancies. To test whether the association between autonomy support and relational wellbeing was mediated by ideal/actual discrepancies, a series of MLM analyses was run with autonomy support and one of the five ideal/actual discrepancies as level 1 explanatory variables (centered within each participant), country as a level 2 explanatory variable, and relational well-being as the outcome. Results are presented in Table 7. Notably, both autonomy support and the various ideal/actual discrepancies (with the exception of extraversion) retained significant associations with relational well-being when both were entered as explanatory variables. That is, both constructs accounted independently for variance in well-being. The typical individual was better off when with autonomy supportive partners, and when his or her actual self-concept was closer to his or her ideal. Country membership had no main effect on well-being. Results of tests of moderation by country were similar to those reported above.

Notably, the magnitude of the coefficients for autonomy support dropped slightly when it was entered simultaneously with ideal/actual self-concept discrepancies. The prediction was that discrepancies would partially mediate the effect of autonomy support on well-being. Sobel tests indicate that the relation of autonomy support to well-being was in fact partially mediated by the respective ideal-relational discrepancies for neuroticism (Sobel statistic $=2.10$, $p<0.05$ ), agreeableness (Sobel statistic $=3.13, p<$ 0.01 ), openness (Sobel statistic $=3.15, p<0.01$ ), and marginally for conscientiousness (Sobel statistic $=1.70$, $p<0.09$ ). (Because extraversion discrepancies no longer accounted for variance in well-being when entered simultaneously with autonomy support, mediation is not a possibility for that Big Five self-concept.) Thus, although for the typical individual perceived autonomy support had a direct association with relational well-being, it appears that part of its association was carried by reduced discrepancies between one's ideal and actual self-concept, again supporting the argument that a reduction in ideal/actual self-concept discrepancies is meaningfully related to well-being and is not simply an artifact of psychological need satisfaction.

\section{Discussion}

The idea that discrepancies between ideal and actual self-concept should be negatively associated with wellbeing is not new (Higgins, 1987; Lecky, 1945; Rogers \& Dymond, 1954). Until the present research, however, it was not known whether this finding would hold when measuring self-concept in terms of the Big Five, whether it would hold across cultures, or whether a within-person process linking self-concept discrepancies and well-being could be identified using a newer data analytic approach such as multilevel modeling. The present research contributes to our understanding of the role of self-concept discrepancies in well-being in each of these three regards. Further, by adopting a self-determination theory (SDT) framework that is generally consistent at a metatheoretical level with the work of Rogers (Joseph \& Linley, 2004; Patterson \& Joseph, 2007), the present research adds to our understanding of the aspects of interpersonal relationships that may either magnify or ameliorate such self-concept discrepancies, with consequences for well-being.

An initial finding of the present study, not specifically hypothesized, was that to a large extent participants from three different countries valued the Big Five in a similar manner. That is, they were likely to report wanting to see themselves as more extraverted, open to experience, agreeable, and conscientious, but as less neurotic than they actually saw themselves. The differences between ideal and actual self-concept were mostly significant. Although not predicted, this finding is noteworthy in that it goes beyond reporting mean levels of traits cross-nationally and provides an initial indication of how those traits are valued in different cultures. The results also provide a basis to make some informal within-country comparisons. Looking at raw means in Table 1, it is clear for example that participants in the three countries all valued conscientiousness highly, and clearly preferred not to think of themselves as neurotic. Another metric for assessing the relative importance of the Big Five in these three countries is the strength of the association of the respective ideal/actual discrepancies with relational well-being (Table 3). In this regard, openness and agreeableness seem to be relatively important, in terms of their functional link with well-being. Although interesting, these findings are circumscribed by the fact that samples in all three countries consisted of university students, who perhaps share more in common than might members of the respective societies in general. 
Table 7. The within-person process relating perceived autonomy support, ideal/actual self-concept discrepancies, country, and their interaction to relational well-being in United States, Russian, and Chinese samples combined.

\begin{tabular}{|c|c|c|}
\hline Explanatory variables (grouped by Big Five self-concept) & Typical individual & $S D$ across individuals \\
\hline Extraversion Discrepancy & -0.11 & 0.34 \\
\hline Autonomy support & $0.84 * *$ & $0.63 * *$ \\
\hline Country 1 & -0.12 & - \\
\hline Country 2 & -0.22 & - \\
\hline Interaction (Country $1 \mathrm{X}$ Extraversion Discrepancy) & 0.00 & - \\
\hline Interaction (Country 2 X Extraversion Discrepancy) & -0.24 & - \\
\hline Interaction (Country $1 \mathrm{X}$ Autonomy support) & $0.60 * *$ & - \\
\hline Interaction (Country $2 \mathrm{X}$ Autonomy support) & $0.22 *$ & - \\
\hline Neuroticism Discrepancy & $-0.25 *$ & $0.82 * * \mathrm{~s}$ \\
\hline Autonomy support & $0.81 * *$ & $0.58 * *$ \\
\hline Country 1 & -0.13 & - \\
\hline Country 2 & -0.24 & - \\
\hline Interaction (Country $1 \mathrm{X}$ Neuroticism Discrepancy) & $-0.89 * *$ & - \\
\hline Interaction (Country 2 X Neuroticism Discrepancy) & $-1.00 * *$ & - \\
\hline Interaction (Country $1 \mathrm{X}$ Autonomy support) & 0.13 & - \\
\hline Interaction (Country $2 \mathrm{X}$ Autonomy support) & -0.15 & - \\
\hline Agreeableness Discrepancy & $-0.55 * *$ & $0.64 * *$ \\
\hline Autonomy support & $0.79 * *$ & $0.60 * *$ \\
\hline Country 1 & -0.13 & - \\
\hline Country 2 & -0.24 & - \\
\hline Interaction (Country $1 \mathrm{X}$ Agreeableness Discrepancy) & -0.14 & - \\
\hline Interaction (Country $2 \mathrm{X}$ Agreeableness Discrepancy) & -0.24 & - \\
\hline Interaction (Country $1 \mathrm{X}$ Autonomy support) & $0.54 * *$ & - \\
\hline Interaction (Country $2 \mathrm{X}$ Autonomy support) & 0.05 & - \\
\hline Openness Discrepancy & $-0.41 * *$ & $0.61 * *$ \\
\hline Autonomy support & $0.79 * *$ & $0.61 * *$ \\
\hline Country 1 & -0.13 & - \\
\hline Country 2 & -0.23 & - \\
\hline Interaction (Country $1 \mathrm{X}$ Openness Discrepancy) & -0.18 & - \\
\hline Interaction (Country $2 \mathrm{X}$ Openness Discrepancy) & 0.09 & - \\
\hline Interaction (Country $1 \mathrm{X}$ Autonomy support) & $0.46^{* *}$ & - \\
\hline Interaction (Country $2 \mathrm{X}$ Autonomy support) & $0.20+$ & - \\
\hline Conscientiousness Discrepancy & $-0.43 * *$ & $0.63 * *$ \\
\hline Autonomy support & $0.83 * *$ & $0.59 * *$ \\
\hline Country 1 & -0.13 & - \\
\hline Country 2 & -0.23 & - \\
\hline Interaction (Country $1 \mathrm{X}$ Conscientiousness Discrepancy) & -0.14 & - \\
\hline Interaction (Country $2 \mathrm{X}$ Conscientiousness Discrepancy) & $-0.36^{*}$ & - \\
\hline Interaction (Country $1 \mathrm{X}$ Autonomy support) & $0.68 * *$ & - \\
\hline Interaction (Country $2 \mathrm{X}$ Autonomy support) & $0.21+$ & - \\
\hline
\end{tabular}

Note: Coefficients for 'Typical individual' are unstandardized. Country $1=$ United States; Country $2=$ Russia; Country 3 (China) is treated as redundant in the analysis, meaning that results for Countries 1 and 2 are with reference to Country 3 . $* p<0.05 ; * *<0.01 ;+p<0.09$.

Turning now to the study's hypotheses, the first concerned the relation between self-concept discrepancies and well-being. For participants in China, Russia, and the United States, experiencing a discrepancy between one's personal ideal self-concept, assessed in terms of the Big Five personality traits, and one's actual self-concept when with various relationship partners, was associated with lower levels of wellbeing in one's relationships. There was limited moderation of this association by country membership, but it is important to point out that this moderation changed only the strength and not the direction of the association: regardless of country membership, larger self-concept discrepancies were associated with lower well-being.

The second prediction, based on SDT, was that being with autonomy supportive partners would be associated with smaller ideal/actual discrepancies. This was indeed the case, in all three countries. There was some moderation by country membership in the case of particular discrepancies, but again this moderation affected only the strength and not the direction of the association.

The study also tested the prediction that support for autonomy, conceptualized within SDT as a basic psychological need, should be associated with greater 
well-being. It was, in all three countries. The study found however that the direct path between autonomy support and well-being was partially mediated through reduced self-concept discrepancies. Again, the importance of this is that it suggests that self-concept discrepancies are in themselves relevant to well-being, and are not simply an artifact of psychological need satisfaction.

\section{Cultural implications}

SDT claims on theoretical grounds that certain psychological needs, including the need for autonomy, are universal. Despite some recent cross-cultural work (Chirkov, Ryan, Kim, \& Kaplan, 2003; Chirkov et al., 2005), this claim has not been tested extensively in empirical research. In contrast, some other theorists have suggested that autonomy support is critical only for those who live in cultures where it is explicitly valued, or for those who themselves personally embrace it (e.g., Markus \& Kitayama, 1991), a proposition which may be called the 'match hypothesis.'

The present study explored the universality of the need for autonomy by testing whether the association between autonomy support and ideal/actual selfconcept discrepancies was moderated by culture, operationalized in terms of country membership. Significant interactions between autonomy support and country membership (for example, such that autonomy support was important for some countries but not all, or was important but in opposite directions in different countries) could be taken as providing support for the match hypothesis. However, although there was some moderation by country membership, such that the effects were stronger in some cases for US and Russian than for Chinese participants, still, the effects were in the same direction in all three countries; for the typical individual, when there was an association autonomy support was related to lower ideal/ actual discrepancies.

Although these findings generally support the SDT view that autonomy is a need that may be universal in nature, this prediction should be tested in other countries with other samples. That country membership moderated the strength of autonomy's association with the outcomes in the present study is important to note, and may reflect the possibility that even an underlying need that is universal may be satisfied in ways that reflect local cultural customs and norms, a process only inadequately captured when using translated measures in different countries. Put differently, the weaker associations in some analyses may be an artifact of the nomothetic nature of the present research, that is, a psychometric or linguistic issue, despite the demonstration of construct comparability through MACs analyses. This suggestion however requires further study.

\section{Clinical implications}

Rogers and Dymond (1954) argued that awareness of a gap between one's current self-view and how one would ideally like to be could serve as the source of some forms of clinically relevant distress (see also Higgins, 1989). The present research provides evidence of a within-person process linking ideal/actual selfconcept discrepancies, measured in terms of the Big Five, with lowered well-being. It is possible that some of the distress experienced by clients seeking counseling or psychotherapy may be due to a perceived gap between the client's current state and his or her ideal state, much as these earlier theoreticians predicted. To the extent that this is true, growth and well-being would seem to lie in the direction of more closely approximating one's ideal view of self. Further, based on the present research, it may be possible to infer that some of a client's self-concept related distress may stem from important relationships that are predominantly controlling rather than autonomy supportive.

These two observations pertain to insight the present research can contribute to understanding the nature of a client's presenting problems. Problem identification is important to the extent that it can inform intervention strategy. In this regard, clinicians can find ways to help their clients explore their personal ideals, creating opportunities for them to integrate those ideals more fully into their self-concept. Further, recent work highlights the role played in attaining important clinical outcomes by an autonomy supportive style among health care providers (Sheldon, Williams, \& Joiner, 2003) and psychotherapists (Zuroff, Koestner, Moskowitz, McBride, Marshall, \& Bagby, 2007). Thus, much as Rogers (1961) advocated the therapeutic value of a style characterized by genuineness, empathy, and unconditional positive regard, in light of the present research clinicians might explore a more autonomy supportive style that could help create a climate that is more conducive to personality integration and the pursuit of one's ideal view of self, on the organismic assumption that growth lies in this direction (Joseph \& Linley, 2004).

Importantly, Rogers (1961) speculated that the same kind of facilitative process should characterize nonprofessional interpersonal relationships, and the present research, which focused on six interpersonal relationships, provides empirical support for this prediction. Accordingly, in addition to embracing an autonomy supportive style themselves, clinicians and other helping professionals (e.g., life coaches) may find it important to help clients explore the nature of their close personal relationships (e.g., Williams et al., 2006), teaching them ways to identify and satisfy basic psychological needs such as the need for autonomy in those relationships in ways that are constructive and that can facilitate pursuit of 
their ideals. These suggestions however require further study.

In particular, it will be important for future research to address the possibility that what changes in the presence of an autonomy supportive counselor or friend is the person's ideal view of self, rather than or perhaps in addition to his or her actual self-concept. Rogers acknowledged this possibility, stating that there was in fact no a priori reason to assume that it is the actual rather than ideal self-concept that changes during the course of therapy (Rogers \& Dymond, 1954). He and colleagues discovered however that typically it was the client's actual self-concept that underwent change, coming closer, in successful therapy, to the client's own self-endorsed ideal. Because the ideal self-concept was in the present research static (it was measured only once), it seems clear that what fluctuated in the presence of autonomy supportive partners was the participants' actual self-concept. With Rogers, however, we acknowledge that the therapeutic qualities of a need-supportive relationship could just as easily alter a person's ideal image of self, bringing a perhaps introjected or otherwise unrealistic view of self more closely into line with a person's true potentiality (for a psychodynamic view, see also Horney, 1937/1999).

\section{Limitations}

There are some notable limitations in the present research. Among them, it is important to point out that the present data are self-report and correlational. For this reason, it is not possible to disentangle the direction of causality. The way people think about themselves may elicit either autonomy supportive or controlling responses from their partners, autonomy support may cause changes in self-concept, or a third variable may be affecting both self-concept and partner responses (or one's perception of them). Experimental and longitudinal studies are needed to clarify these issues. In addition, as noted, this research included samples from only three countries, and measured culture only in terms of country membership. To provide a fuller test of the cross-cultural generalizability of these findings it will be necessary to investigate samples from other countries and to use other measures of culture (e.g., Oyserman, Coon, \& Kemmelmeier, 2002; Singelis, 1994; Triandis \& Gelfand, 1998).

\section{Summary}

The present study confirmed the existence of a withinperson process linking discrepancies between ideal and actual self-concept, assessed in terms of the Big Five personality traits, with decrements to well-being.
Further, ideal/actual discrepancies were smaller when participants were with autonomy supportive partners. Importantly, these findings held in three different countries, suggesting that there may be something universally important in being the person one would like to be, and that relationships that support the need for autonomy may play an important role in facilitating this outcome.

\section{Acknowledgments}

The authors wish to thank Tatiana Strabakhina from Novgorod State University and Yan Rongshuang from Shandong Normal University for their collaboration and consultation during the completion of this study. The data for this study were collected for the dissertation of the first author.

\section{Note}

1. Although we did not have specific predictions about how country membership would moderate the relation between self-concept discrepancies and well-being, we considered it important to test for moderation by country in an exploratory mode. In this way, analyses would also parallel those for autonomy support, about which we did have specific predictions regarding moderation.

\section{References}

Bostic, T.J., Rubio, D., \& Hood, M. (2000). A validation of the subjective vitality scale using structural equation modeling. Social Indicators Research, 52, 313-324.

Byrne, B. (2001). Structural equation modeling with AMOS: Basic concepts, applications, and programming. Mahwah, NJ: Lawrence Erlbaum.

Byrne, B. (2002). Validating the measurement and structure of self-concept: Snapshots of past, present, and future research. American Psychologist, 57, 897-909.

Cheung, G.W., \& Rensvold, R.B. (2000). Assessing extreme and acquiescence response sets in cross-cultural research using structural equations modeling. Journal of CrossCultural Psychology, 31, 187-212.

Chirkov, V.I., Ryan, R.M., Kim, Y., \& Kaplan, U. (2003). Differentiating autonomy from individualism and independence: A self-determination theory perspective on internalization of cultural orientations and well-being. Journal of Personality and Social Psychology, 84, 97-110.

Chirkov, V., Ryan, R.M., \& Willness, C. (2005). Cultural context and psychological needs in Canada and Brazil: Testing a self-determination approach to the internalization of cultural practices, identity, and well-being. Journal of Cross-Cultural Psychology, 36, 423-443.

Deci, E.L., La Guardia, J.G., Moller, A.C., Scheiner, M.J., \& Ryan, R.M. (2006). On the benefits of giving as well as receiving autonomy support: Mutuality in close friendships. Personality and Social Psychology Bulletin, 32, 313-327. 
Deci, E.L., \& Ryan, R.M. (2000). The 'what' and 'why' of goal pursuits: Human needs and the self-determination of behavior. Psychological Inquiry, 11, 227-268.

Fleeson, W. (2007). Studying personality processes: Explaining change in between-persons longitudinal and within-person multilevel models. In R.W. Robins, R.C. Fraley, \& R.F. Krueger (Eds.), Handbook of research methods in personality psychology (pp. 523-542). New York: Guilford.

Grolnick, W.S., \& Ryan, R.M. (1989). Parent styles associated with children's self-regulation and competence in school. Journal of Educational Psychology, 81, 143-154.

Higgins, E.T. (1987). Self-discrepancy: A theory relating self and affect. Psychological Review, 94, 319-340.

Higgins, E.T. (1989). Self-discrepancy theory: What patterns of self-beliefs cause people to suffer? Advances in Experimental Social Psychology, 22, 93-136.

Horney, K. (1999). The neurotic personality of our time. London: Routledge, Original work published 1937.

Jang, H., Reeve, J., Ryan, R.M., \& Kim, A. (in press). Can self-determination theory explain what underlies the productive, satisfying learning experiences of collectivistically-oriented Korean students? Journal of Educational Psychology.

John, O.P., \& Srivastava, S. (1999). The Big Five trait taxonomy: History, measurement, and theoretical perspectives. In L.A. Pervin, \& O.P. John (Eds.), Handbook of personality: Theory and research (2nd ed., pp. 102-138). New York: Guilford.

Joseph, S., \& Linley, P.A. (2004). Positive therapy: A positive psychological theory of therapeutic practice. In P.A. Linley, \& S. Joseph (Eds.), Positive psychology in practice (pp. 354-368). Hoboken, NJ: Wiley.

La Guardia, J.G., Ryan, R.M., Couchman, C.E., \& Deci, E.L. (2000). Within-person variation in security of attachment: A self-determination theory perspective on attachment, need fulfillment, and well-being. Journal of Personality and Social Psychology, 79, 367-384.

Lecky, P. (1945). Self-consistency: A theory of personality. New York: Island.

Little, T.D. (1997). Mean and covariance structures (MACS) analyses of cross-cultural data: Practical and theoretical issues. Multivariate Behavioral Research, 32, 53-76.

Little, T.D. (2000). On the comparability of constructs in cross-cultural research: A critique of Cheung and Rensvold. Journal of Cross-Cultural Psychology, 31, 213-219.

Little, T.D., Lindenberger, U., \& Nesselroade, J.R. (1999). On selecting indicators for multivariate measurement and modeling with latent variables: When 'good' indicators are bad and 'bad' indicators are good. Psychological Methods, 4, 192-211.

Lynch, M.F., Plant, R.W., \& Ryan, R.M. (2005). Psychological needs and threat to safety: Implications for staff and patients in a psychiatric hospital for youth. Professional Psychology, 36, 415-425.

Magnusson, D., \& Endler, N.S. (1977). Personality at the crossroads: Current issues in interactional psychology. Hillsdale, NJ: Erlbaum.

Markland, D., Ryan, R.M., Tobin, V.J., \& Rollnick, S. (2005). Motivational interviewing and self-determination theory. Journal of Social and Clinical Psychology, 24, 811-831.

Markus, H.R., \& Kitayama, S. (1991). Culture and the self: Implications for cognition, emotion, and motivation. Psychological Review, 98, 224-253.

McCrae, R.R., \& Allik, J. (Eds.). (2002). The Five-Factor model of personality across cultures. New York: Kluwer.

McCrae, R.R., Costa, P.T. Jr, \& (2003). Personality in adulthood: a five-factor theory perspective. New York: The Guilford Press.

McCrae, R.R., Martin, T.A., Oryol, V.E., Rukavishnikov, A.A., Senin, I.G., et al. (2004). Consensual validation of personality traits across cultures. Journal of Research in Personality, 38, 179-201.

McCrae, R.R., \& Terracciano, A. (2005). Personality profiles of cultures: Aggregate personality traits. Journal of Personality and Social Psychology, 89, 407-425.

Oyserman, D., Coon, H.M., \& Kemmelmeier, M. (2002). Rethinking individualism and collectivism: Evaluation of theoretical assumptions and meta-analyses. Psychological Bulletin, 128, 3-72.

Patrick, H., Knee, C.R., Canevello, A., \& Lonsbary, C. (2007). The role of need fulfillment in relationship functioning and well-being: A self-determination theory perspective. Journal of Personality and Social Psychology, 92, 434-457.

Patterson, P.G., \& Joseph, S. (2007). Person-centered personality theory: Support from self-determination theory and positive psychology. Journal of Humanistic Psychology, 47, 117-139.

Reeve, J. (2002). Self-determination theory applied to educational settings. In E.L. Deci, \& R.M. Ryan (Eds.), Handbook of self-determination research (pp. 183-203). Rochester, NY: University of Rochester Press.

Reeve, J., Bolt, E., \& Cai, Y. (1999). Autonomy-supportive teachers: How they teach and motivate students. Journal of Educational Psychology, 91, 537-548.

Roberts, B.W., Robins, R.W., Caspi, A., \& Trzesniewski, K.H. (2003). Personality trait development in adulthood. In J.L. Mortimer, \& M. Shanahan (Eds.), Handbook of the life course (pp. 579-596). New York: Plenum Press.

Rogers, C.R. (1961). On becoming a person: a therapist's view of psychotherapy. Boston: Houghton Mifflin.

Rogers, C.R., \& Dymond, R.F. (Eds.). (1954). Psychotherapy and personality change: co-ordinated research studies in the client-centered approach. Chicago: University of Chicago Press.

Rudy, D., Sheldon, K., Awong, T., \& Tan, H. (2007). Autonomy, culture, and well-being: The benefits of inclusive autonomy. Journal of Research in Personality, 41, 983-1007.

Ryan, R.M., \& Deci, E.L. (2000). Self-determination theory and the facilitation of intrinsic motivation, social development, and well-being. American Psychologist, 55, 68-78.

Ryan, R.M., \& Frederick, C. (1997). On energy, personality, and health: Subjective vitality as a dynamic reflection of well-being. Journal of Personality, 65, 529-565.

Ryan, R.M., \& Lynch, M.F. (2003). Motivation and classroom management. In R. Curren (Ed.), Blackwell companions to philosophy: A companion to the philosophy of education (pp. 260-271). New York: Blackwell. 
Schmitt, D.P., Allik, J., Mccrae, R.R., \& Benet-Martinez, V. (2007). The geographic distribution of big five personality traits: Patterns and profiles of human self-description across 56 nations. Journal of Cross-Cultural Psychology, $38,173-212$.

Sheldon, K.M., Ryan, R.M., Rawsthorne, L.J., \& Ilardi, B. (1997). Trait self and true self: Cross-role variation in the Big-Five personality traits and its relations with psychological authenticity and subjective well-being. Journal of Personality and Social Psychology, 73, 1380-1393.

Sheldon, K.M., Williams, G., \& Joiner, T. (2003). Self-determination theory in the clinic: Motivating physical and mental health. New Haven: Yale University Press.

Shoda, Y., Mischel, W., \& Wright, J.C. (1994). Intraindividual stability in the organization and patterning of behavior: Incorporating psychological situations into the idiographic analysis of personality. Journal of Personality and Social Psychology, 67, 674-687.

Singelis, T.M. (1994). The measurement of independent and interdependent self-construals. Personality \& Social Psychology Bulletin, 20, 580-591.

Srivastava, S., John, O.P., Gosling, S.D., \& Potter, J. (2003). Development of personality in early and middle adulthood: Set like plaster or persistent change? Journal of Personality and Social Psychology, 84, 1041-1053.
Triandis, H.C., \& Gelfand, M.J. (1998). Converging measurement of horizontal and vertical individualism and collectivism. Journal of Personality and Social Psychology, 74, 118-128.

Vansteenkiste, M., Zhou, M., Lens, W., \& Soenens, B. (2005). Experiences of autonomy and control among Chinese learners: Vitalizing or immobilizing? Journal of Educational Psychology, 96, 755-764.

Watson, D., Clark, L.A., \& Tellegen, A. (1988). Development and validation of brief measures of positive and negative affect: The PANAS scales. Journal of Personality \& Social Psychology, 54, 1063-1070.

Williams, G.C., \& Deci, E.L. (2001). Activating patients for smoking cessation through physician autonomy support. Medical Care, 39, 813-823.

Williams, G.C., Lynch, M.F., McGregor, H., Ryan, R.M., Sharp, D., \& Deci, E.L. (2006). Validation of the important other climate questionnaire: Assessing autonomy support for health related change. Families, Systems, and Health, 24, 179-194.

Zuroff, D.C., Koestner, R., Moskowitz, D.S., McBride, C., Marshall, M., \& Bagby, M. (2007). Autonomous motivation for therapy: A new common factor in brief treatments for depression. Psychotherapy Research, 17, 137-148. 